\title{
APLICAÇÃO DE PROGRAMA COMPUTACIONAL LIVRE EM PLANEJAMENTO DE EXPERIMENTOS: UM TUTORIAL
}

Fabíola Manhas Verbi Pereira ${ }^{\mathrm{a}}$ e Edenir Rodrigues Pereira-Filho ${ }^{\mathrm{b}, *}$

anstituto de Química, Universidade Estadual Paulista, 14800-060 Araraquara - SP, Brasil

bDepartamento de Química, Universidade Federal de São Carlos, 13565-905 São Carlos - SP, Brasil

Recebido em 24/03/2018; aceito em 06/06/2018; publicado na web em 28/06/2018

\begin{abstract}
APPLICATION OF FREE COMPUTATIONAL PROGRAM IN EXPERIMENTAL DESIGN: A TUTORIAL. This tutorial intends to show to the readers a step-by-step guide to perform a factorial design in their experiments. The examples used here cover most of doubts and questions related to teaching classes at graduation course. Free computational software was applied, as well as, codes were designed by the authors for the calculations. The complexity of each example is attached to the challenges and decisions that the experimentalist faces in its research. In addition, 24 videos were prepared to help the readers. The authors hope to foster the factorial design as an easy and helpful tool for chemists and general researchers in academia and industry.
\end{abstract}

Keywords: Octave; factorial design; analysis of variance; regression model; template surface.

\section{INTRODUÇÃO}

A aplicação de ferramentas quimiométricas relacionadas ao planejamento fatorial de experimentos é uma realidade em diversos laboratórios de pesquisa e também no setor industrial. ${ }^{1,2}$ Tais ferramentas têm contribuído para aprimorar processos de fabricação e de síntese, bem como na proposição rápida de métodos analíticos mais sensíveis e com alta frequência de amostragem. ${ }^{3-9}$ As vantagens da aplicação do planejamento fatorial são inquestionáveis e amplas, podendo ser resumidas em: (1) execução de um número reduzido de experimentos quando comparado com o procedimento univariado convencional; (2) economia de recursos financeiros; (3) obtenção de resultados com maiores confiabilidades química e estatística; (4) possibilidade de obter um modelo matemático que permitirá a realização de previsões em condições que não foram testadas. ${ }^{10-25}$

$\mathrm{Na}$ literatura científica nacional e internacional é frequente encontrar relatos de inúmeras aplicações, além de quase uma dezena de revisões, livros e tutoriais sobre o assunto. ${ }^{26-48}$ Adicionalmente, desde 2015 vários livros em língua portuguesa ${ }^{25,45}$ têm sido publicados sobre o tema, agregando mais conhecimento à produção pioneira publicada por Barros Neto et $a l .{ }^{48}$ Estes aspectos mostram a popularidade, a importância e a consolidação do tema. Entretanto, apesar de todos os esforços oriundos de diferentes autores brasileiros na proposição de experimentos didáticos para o ensino de planejamento fatorial, um tópico ainda precisa ser complementado: a utilização de programas livres e de fácil acesso, além da viabilização e da popularização do emprego de tais ferramentas pelos usuários. ${ }^{49-51}$ Novaes et al. ${ }^{52}$ publicaram em 2017 uma revisão abordando a utilização de planejamento fatorial na otimização de variáveis de processo. Os autores efetuaram uma excelente compilação dos principais programas comerciais disponíveis e apontaram também a faixa de preços dos mesmos. Entretanto, exemplos que utilizam planejamento fatorial e programas livres são inexistentes na literatura científica nacional e também internacional.

Assim, a principal proposta deste tutorial é apresentar a utilização do programa livre Octave ${ }^{53}$ como uma ferramenta de fácil implementação em estudos que envolvam planejamento fatorial. Desta forma, foram propostas três rotinas computacionais direcionadas para:

*e-mail: erpf@ufscar.br
(1) efetuar os cálculos de efeitos em um planejamento fatorial completo e contrastes para um planejamento fracionário, (2) proposição de modelos de regressão de primeiro ou segundo grau e (3) visualização de superfície de resposta e gráfico de contorno. Além disso, foi desenvolvido um arquivo no Microsoft Excel para inspeção visual das superfícies de respostas geradas. A Figura 1 mostra um esquema geral para a utilização deste tutorial onde são abordadas 6 etapas que se complementam: (1) Triagem das variáveis, (2) Proposição de modelos, (3 e 4) Analysis of Variance (Tabela Anova), (5) Avaliação dos coeficientes e (6) Validação do modelo proposto. O esquema da Figura 1 será discutido detalhadamente nas próximas seções deste tutorial.

O programa Octave é livre podendo ser utilizado em diferentes sistemas operacionais, tais como Windows, Linux, Mac, BSD e Source, sendo possível o seu emprego em duas versões: Comand line interface (CLI) e Guide user interface (GUI). São escassos os trabalhos que já empregaram esse programa na literatura com objetivos didáticos ou até mesmo científicos. Assim, esse tutorial tem como principal objetivo preencher essa lacuna por meio do uso do programa Octave com a aplicação de três rotinas computacionais e quatro exemplos coletados na literatura. $\mathrm{Na}$ apresentação dos exemplos também foram preparados vídeos para auxiliar o leitor na utilização do programa e das ferramentas aqui ilustradas.

\section{PARTE EXPERIMENTAL}

\section{Descrição dos conjuntos de dados}

Para a ilustração das ferramentas que são tratadas neste tutorial foram selecionados três conjuntos de dados químicos a partir de estudos publicados no periódico científico Journal of The Brazilian Chemical Society. ${ }^{54-56}$ A autorização para uso destes dados foi gentilmente cedida pela PubliSBQ. A particularidade dos artigos escolhidos está relacionada com a possibilidade de exemplificar 4 estratégias de utilização de planejamento fatorial na análise de experimentos: (1) planejamento fatorial completo, (2) fracionário e planejamentos para geração de modelos de regressão constituídos por matrizes do tipo (3) Doehlert ${ }^{42}$ e (4) Box-Behnken. ${ }^{4}$ Os exemplos $1 \mathrm{e}$ 2 estão relacionados com a etapa 1 mostrada na Figura 1 e ilustram a triagem das variáveis mais importantes para o estudo de um determinado problema. A proposta é apresentar uma sequência lógica 
PROCEDIMENTO PARA REALIZACÃO E INTERPRETACÃO DE UM PLANEJAMENTO FATORIAL

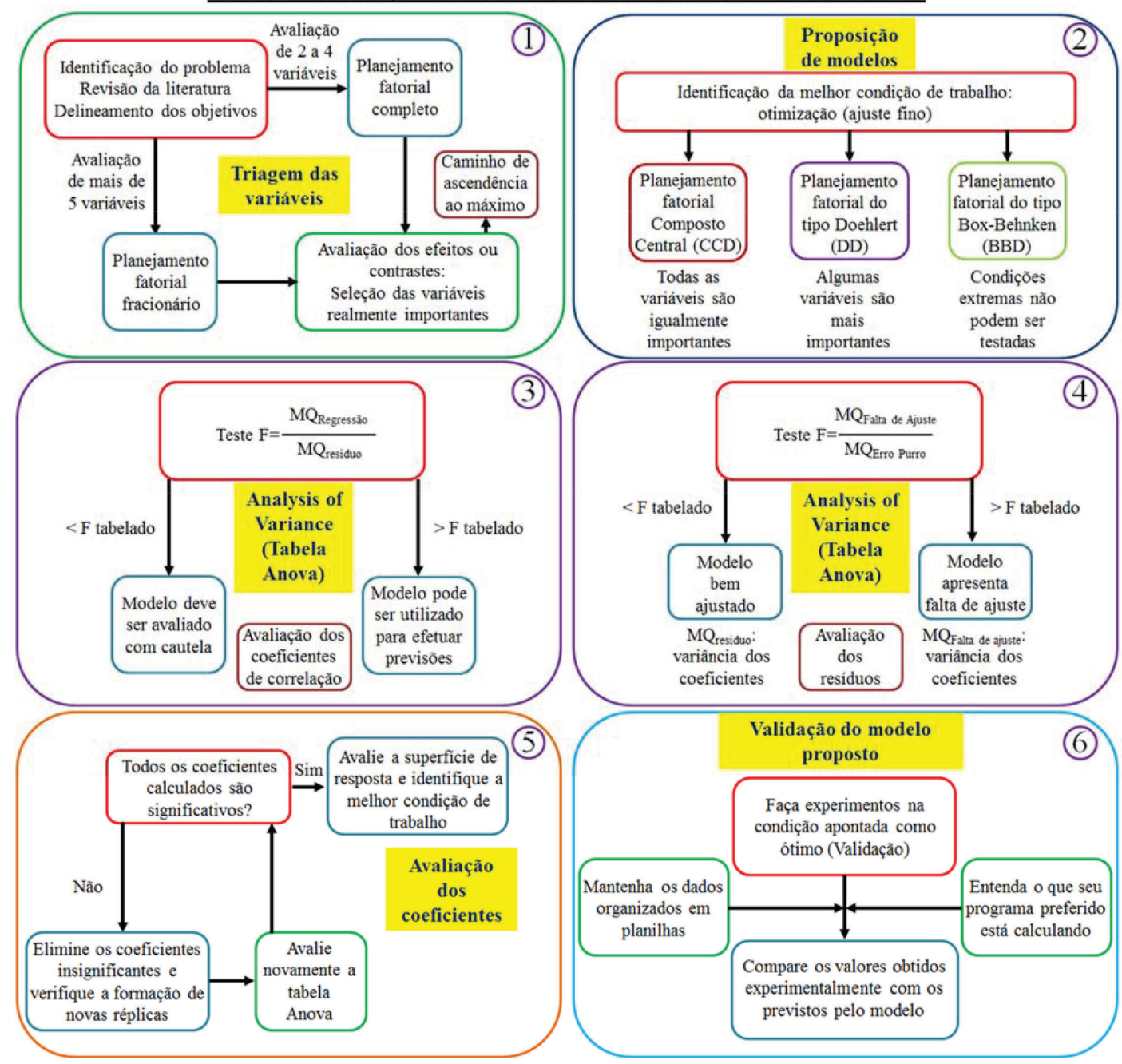

Figura 1. Procedimento geral composto por 6 etapas para a realização e interpretação correta de um planejamento de experimentos

com o aumento do grau de complexidade e de refinamento que cada conjunto de dados pode oferecer. Assim, os exemplos 3 e 4 mostram a utilização das etapas de 3 a 6 da Figura 1.

O exemplo 1 aborda a necessidade em melhorar as condições experimentais de um procedimento para a determinação de espécies de antimônio inorgânico. Três variáveis foram avaliadas com o planejamento fatorial completo de 2 níveis $\left(2^{3}\right){ }^{54}$

Já no exemplo 2, é mostrado um planejamento fatorial fracionário de 2 níveis com o objetivo de testar a influência de 4 variáveis. A geratriz utilizada foi 1234 , onde a quarta variável foi obtida pela multiplicação das variáveis 1,2 e $3\left(2^{4-1}\right)$. O objetivo geral foi a caracterização de micropartículas quanto ao seu diâmetro médio e à distribuição do tamanho. ${ }^{55}$

Para demonstrar o refinamento do resultado obtido pelo planejamento fatorial completo do exemplo 1, retornamos ao primeiro conjunto de dados, ${ }^{54}$ porém, utilizando um planejamento do tipo Doehlert. Assim, no exemplo 3 foi empregado o arquivo "template_superfície" preparado no Microsoft Excel para visualização da superfície de resposta e identificação da melhor condição de trabalho. Para aqueles que não possuem o Microsoft Excel, foi preparada uma rotina computacional chamada "super_fabi" para visualização da superfície de resposta e gráfico de contorno.

O exemplo 4 mostra a utilização do planejamento Box-Behnken para avaliar a conversão de álcool benzílico, tendo como resposta a ser monitorada o rendimento da formação de benzaldeído. ${ }^{56}$ Esse quarto exemplo contou com 4 variáveis e a melhor condição de trabalho proposta pelo modelo de regressão foi identificada com a ferramenta Solver disponível no Microsoft Excel e com a realização de derivadas parciais no Octave ou no Excel.

\section{Rotinas computacionais utilizadas}

No desenvolvimento deste tutorial e cálculos dos exemplos utilizados, foram preparadas três rotinas computacionais no programa Octave (versão 4.2.1). ${ }^{53}$ A primeira rotina é denominada de "fabi_efeito" e tem como objetivo calcular os efeitos, a porcentagem de importância relativa de cada efeito ${ }^{57}$ e seus erros. ${ }^{25,58} \mathrm{O}$ material suplementar (Rotina computacional fabi_efeito) mostra a rotina preparada e a mesma pode ser utilizada em planejamentos completos e também fracionários com ou sem a realização de réplicas.

A segunda rotina computacional é denominada "regression2" e permite o cálculo da tabela contendo a análise de variância (Analysis of variance, Anova). Além disso, são gerados gráficos mostrando as médias quadráticas da regressão, resíduo, erro puro e falta de ajuste, bem como os testes $\mathrm{F}$ (calculados e tabelados), valores de coeficientes de regressão (variação explicada e explicável), valores experimentais versus previstos, valores previstos versus resíduos, histograma dos resíduos e coeficientes de regressão calculados. 
Essa rotina computacional também é apresentada no material suplementar (Rotina computacional regression2). A terceira rotina ("super_fabi”) foi preparada para mostrar superfície de resposta e gráfico de contorno dos modelos calculados.

As rotinas podem ser utilizadas também no programa computacional Matlab sem necessidade de qualquer adaptação.

\section{RESULTADOS E DISCUSSÃO}

\section{Exemplo 1: Planejamento fatorial completo}

No primeiro exemplo sobre o uso de planejamento fatorial completo, os autores ${ }^{54}$ estudaram a influência de 3 variáveis (planejamento $2^{3}$ ) na intensidade do sinal de fluorescência visando a determinação de espécies de antimônio. A Tabela 1 mostra os experimentos realizados, bem como as respostas obtidas. Ao todo foram executados 11 experimentos ( 8 experimentos mais 3 réplicas no ponto central), o objetivo era obter uma alta intensidade de fluorescência e realizar uma triagem das variáveis mais importantes (etapa 1 da Figura 1).

É recomendável que a organização dos dados deva ser realizada em uma planilha no Excel, facilitando a manipulação dos mesmos. Alternativamente, a organização dos dados pode ser efetuada no próprio Octave e oportunamente serão mostrados vídeos de como preparar os dados neste programa.

Com os experimentos descritos na Tabela 1 é possível calcular 7 efeitos: 3 principais, sendo um para cada variável de forma individual (1, 2 e 3), 3 de interações secundárias $(12,13$ e 23) e 1 de interação de terceira ordem (123). Assim, é necessário montar uma matriz (X) de dados para o cálculo desses 7 efeitos. Os efeitos 1, 2 e 3 serão calculados por meio das colunas codificadas mostradas na Tabela 1 . Já os efeitos secundários e o de terceira ordem serão calculados ao multiplicar as respectivas colunas. Por exemplo, a interação 12 (interação entre as variáveis 1 e 2) será calculada ao multiplicar as colunas das variáveis 1 e 2 e assim por diante. ${ }^{25}$ Estas operações podem ser facilmente efetuadas no Excel ou no próprio Octave e a matriz $\mathbf{X}$ formada terá as dimensões 8:7, onde 8 representa os experimentos de 1 a 8 (Tabela 1) e 7 os efeitos que serão calculados. Os experimentos no ponto central não precisam ser utilizados no cálculo dos efeitos.

Como os autores efetuaram 3 réplicas no ponto central (experimentos de 9 a 11 na Tabela 1) é possível calcular a variância, o erro experimental, o erro de um efeito, bem como o valor de $t$ correspondente. ${ }^{48}$ Para o primeiro exemplo temos que:

Variância Experimental $=\frac{\sum\left(x_{i}-\bar{x}\right)^{2}}{n-1}=\frac{(137,5-137)^{2}+(137,5-137)^{2}+(137,8-137)^{2}}{3-1}=1,29$ em que $\bar{x}$ é a média dos 3 experimentos no ponto central (experimentos de 9 a 11). Para o erro experimental teremos:

$$
\text { Erro experimental }=\sqrt{\frac{\sum\left(x_{i}-\bar{x}\right)^{2}}{n-1}}=\sqrt{1,29}=1,14
$$

Como foram efetuadas 3 réplicas $(n=3)$, o número de graus de liberdade será 2 ( $\mathrm{n}-1)$ e o correspondente valor de $t$ com $95 \%$ de confiança será 4,30 . Todos os exemplos desse tutorial e as rotinas computacionais preparadas utilizaram um intervalo de confiança de 95\%. O erro de um efeito ${ }^{52}$ é calculado pela Equação 3:

$$
\text { Erro de um efeito }=\frac{2 \times \text { Erro experimental }}{\sqrt{n \times 2^{k}}}
$$

em que $\mathrm{n}$ e $\mathrm{k}$ são o número de réplicas efetuadas e o número de variáveis, respectivamente.

Para o exemplo 1 teremos:

$$
\text { Erro de um efeito }=\frac{2 \times 1,14}{\sqrt{3 \times 2^{3}}}=0,46
$$

Para proceder os cálculos, no Octave deve ser digitada a seguinte linha de comando:

[efeito, porc]=fabi_efeito $(\mathrm{X}, \mathrm{y}, 0.46,4.30)$;

em que X, y, 0.46 e 4.30 representam, respectivamente, a matriz $\mathbf{X}$ (8:7), o vetor y (8:1) com a resposta (intensidade de fluorescência), o erro de um efeito e o valor de $t$ correspondente ao número de graus de liberdade. Os três experimentos replicados no ponto central não precisam ser considerados. Assim, a matriz $\mathbf{X}$ terá 8 linhas no lugar de 11. Os parâmetros de saída (outputs) "efeito"e "porc" mostram os efeitos calculados e a porcentagem para cada um deles. Caso os experimentos não tenham réplicas, não será possível calcular os erros experimental e de um efeito, assim o operador deve substituir o erro de um efeito e o valor de $t$ pelo valor zero ([efeito, porc, erro_efeito]=fabi_efeito $(\mathrm{X}, \mathrm{y}, 0,0)$;)

A rotina apresentará os gráficos mostrados na Figura 2. O primeiro gráfico (Porcentagem dos efeitos, Figura 2a) mostra a porcentagem que cada efeito exerce sobre o total dos efeitos ${ }^{57}$ e foi calculada por meio da Equação 4:

$$
\text { Porcentagem de um efeito }=\frac{\text { Efeito }_{i}}{\sum\left(\text { Efeitos }^{2}\right)} \times 100
$$

É possível observar que três efeitos representam grande

\begin{tabular}{|c|c|c|c|c|c|c|c|}
\hline \multirow{2}{*}{ Experimentos } & \multicolumn{2}{|c|}{$\mathrm{HCl}(\mathrm{v} 1)$} & \multicolumn{2}{|c|}{$\mathrm{NaBH}_{4}(\mathrm{v} 2)$} & \multicolumn{2}{|c|}{ Tempo de retenção (v3) } & \multirow{2}{*}{$\begin{array}{l}\text { Intensidade de } \\
\text { fluorescência do } \mathrm{Sb}\end{array}$} \\
\hline & Codificado & Real (mol/L) & Codificado & $\operatorname{Real}(\%, \mathrm{~m} / \mathrm{v})$ & Codificado & Real (min) & \\
\hline 1 & -1 & 3 & -1 & 1 & -1 & 10 & 178,4 \\
\hline 2 & -1 & 3 & -1 & 1 & 1 & 30 & 167,5 \\
\hline 3 & -1 & 3 & 1 & 3 & -1 & 10 & 225,7 \\
\hline 4 & -1 & 3 & 1 & 3 & 1 & 30 & 218,1 \\
\hline 5 & 1 & 5 & -1 & 1 & -1 & 10 & 86,6 \\
\hline 6 & 1 & 5 & -1 & 1 & 1 & 30 & 91,0 \\
\hline 7 & 1 & 5 & 1 & 3 & -1 & 10 & 195,6 \\
\hline 8 & 1 & 5 & 1 & 3 & 1 & 30 & 189,2 \\
\hline 9 & 0 & 4 & 0 & 2 & 0 & 20 & 137,5 \\
\hline 10 & 0 & 4 & 0 & 2 & 0 & 20 & 135,7 \\
\hline 11 & 0 & 4 & 0 & 2 & 0 & 20 & 137,8 \\
\hline
\end{tabular}
importância relativa quando comparados com os demais. Esses efeitos são aqueles identificados com os números 2 (variável 2),

Tabela 1. Planejamento fatorial completo $2^{3}$ para verificar a influência de 3 variáveis (v1 a v3) na intensidade de fluorescência do Sb. 
(a)

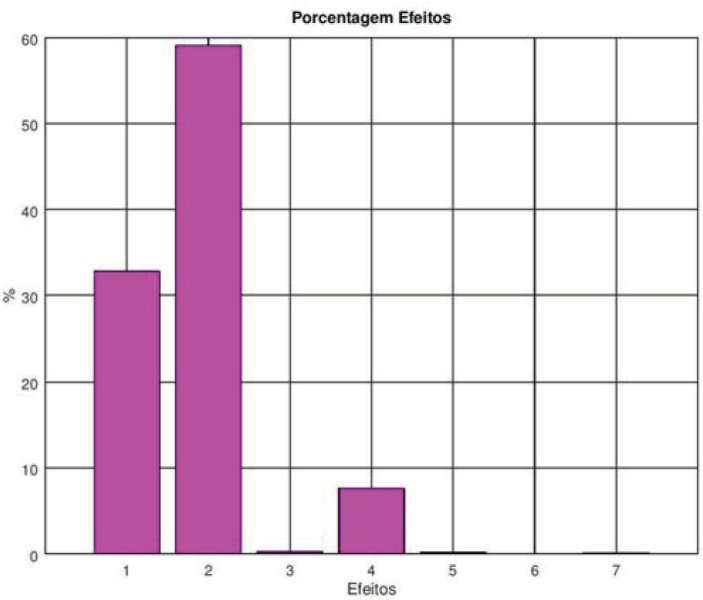

(b)

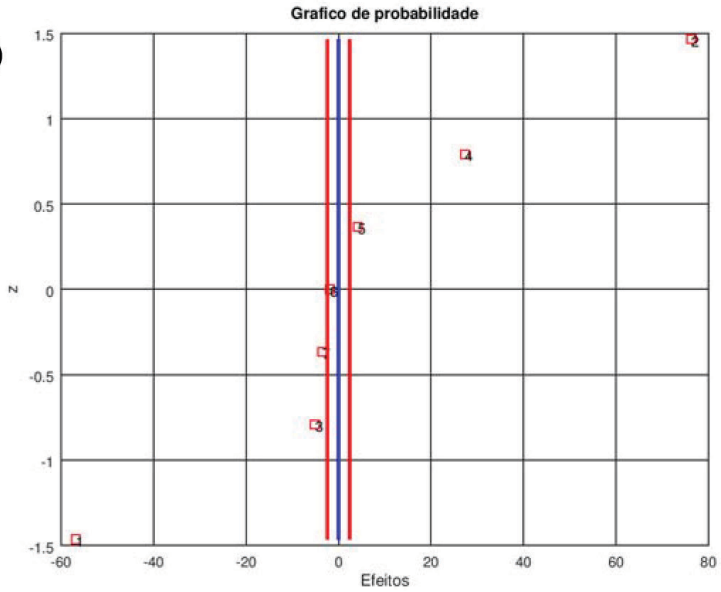

Figura 2. Porcentagem relativa dos efeitos calculados (a) e o gráfico de probabilidade (b) para o exemplo 1

1 (variável 1) e 4 (interação 12) e apresentam, nesta ordem, maior grau de importância. Já o segundo gráfico (Gráfico de probabilidade, Figura 2b) é possível confirmar que esses 3 efeitos são realmente os mais importantes, pois estão posicionados a certa distância do 0 no eixo x (Efeitos). A linha azul vertical mostra a posição do zero no eixo $\mathrm{x}$ e as duas linhas verticais vermelhas indicam o intervalo de confiança ${ }^{25,48}$ dos efeitos calculados por meio da Equação 3:

\section{Intervalo de confiança dos efeitos $=$ erro de um efeito $\times t$}

No gráfico de probabilidade é notado que os efeitos 2 (relativo à variável 2), 1 (relativo à variável 1) e 4 (relativo à interação 12) estão distantes do intervalo de confiança dos efeitos, delimitado pelas duas linhas verticais vermelhas. Além disso, nota-se também que o efeito da variável 3 (identificado com o número 3) e suas interações 13, 23 e 123, identificados com os números 5, 6 e 7, respectivamente, não são importantes, estando localizadas próximas ao zero no eixo x. Assim, a variável 3 pode ser removida do planejamento fatorial, resultando então em novas réplicas e permitindo calcular um novo valor para o erro de um efeito. Essa operação foi realizada e é mostrada no material suplementar (Resultados obtidos para o exemplo 1 após a remoção da variável 3 ).

Como conclusão do exemplo 1, é possível notar que para obter altos sinais de fluorescência é necessário, idealmente, diminuir e aumentar as condições experimentais das variáveis 1 e 2, respectivamente. Já a variável 3 foi inócua para a faixa testada de 10 a 30 min, podendo ser fixada em qualquer condição dentro deste intervalo.

\section{Planejamento fatorial fracionário}

O exemplo 2 mostra a aplicação da rotina "fabi_efeito" no cálculo de contrastes ${ }^{25,48}$ de um planejamento fatorial fracionário e está relacionada também com a etapa 1 descrita na Figura 1. A Tabela 2 mostra os experimentos efetuados e as respostas para o tamanho do diâmetro de partícula (resposta 1) e distribuição dos mesmos (resposta 2). O objetivo dos autores ${ }^{55}$ foi minimizar e maximizar as respostas 1 e 2 , respectivamente.

Os autores efetuaram 3 réplicas no ponto central (variáveis codificadas em 0 , experimentos 9 - 11) e a quarta variável foi obtida pela multiplicação das colunas das variáveis 1, 2 e 3, sendo a geratriz igual a 1234. Com as réplicas no ponto central é possível calcular o erro de um efeito para ambas as respostas: 0,62 e 0,059 para as respostas 1 e 2, respectivamente, como mostram as Equações de 1 a 3. Com a configuração desse planejamento fatorial fracionário, os efeitos de primeira ordem estão confundidos com efeitos de terceira ordem e os efeitos de segunda ordem estão confundidos entre si..$^{25,48}$ Com os 11 experimentos efetuados é possível calcular 7 contrastes (C): 1+234 (C1), 2+134 (C2), 3+124 (C3), 4+123 (C4), 12+34 (C5), $13+24$ (C6) e 14+23 (C7).

Tabela 2. Planejamento fatorial fracionário $2^{4-1}$ para verificar a influência de 4 variáveis (v1 a v4) em duas respostas

\begin{tabular}{|c|c|c|c|c|c|c|c|c|c|c|}
\hline \multirow[t]{2}{*}{ Experimentos } & \multicolumn{2}{|c|}{$\begin{array}{l}\text { Massa de polietileno } \\
\text { glicol (PEG) (v1) }\end{array}$} & \multicolumn{2}{|c|}{$\begin{array}{l}\text { Volume da fase aquosa } \\
\text { (v2) }\end{array}$} & \multicolumn{2}{|c|}{$\begin{array}{l}\text { Porcentagem de álcool po- } \\
\text { livinílico (PVA) (v3) }\end{array}$} & \multicolumn{2}{|c|}{$\begin{array}{l}\text { Volume da fase aquosa } \\
\qquad(\mathrm{v} 4=1234)\end{array}$} & \multicolumn{2}{|c|}{ Respostas monitoradas } \\
\hline & Codificado & Real (mg) & Codificado & Real (mL) & Codificado & Real (\%) & Codificado & Real (mL) & Diâmetro $(\mu \mathrm{m})$ & Distribuição \\
\hline 1 & 1 & 200 & -1 & 1 & -1 & 0,5 & 1 & 100 & 28,4 & 2,02 \\
\hline 2 & -1 & 50 & -1 & 1 & 1 & 2 & 1 & 100 & 26,0 & 1,29 \\
\hline 3 & 1 & 200 & 1 & 3 & 1 & 2 & 1 & 100 & 14,1 & 1,62 \\
\hline 4 & -1 & 50 & 1 & 3 & -1 & 0,5 & 1 & 100 & 8,1 & 2,51 \\
\hline 5 & -1 & 50 & 1 & 3 & 1 & 2 & -1 & 30 & 13,0 & 4,76 \\
\hline 6 & -1 & 50 & -1 & 1 & -1 & 0,5 & -1 & 30 & 14,7 & 1,79 \\
\hline 7 & 1 & 200 & -1 & 1 & 1 & 2 & -1 & 30 & 7,8 & 3,23 \\
\hline 8 & 1 & 200 & 1 & 3 & -1 & 0,5 & -1 & 30 & 6,7 & 3,61 \\
\hline 9 & 0 & 125 & 0 & 2 & 0 & 1,25 & 0 & 65 & 20,8 & 1,56 \\
\hline 10 & 0 & 125 & 0 & 2 & 0 & 1,25 & 0 & 65 & 18,6 & 1,35 \\
\hline 11 & 0 & 125 & 0 & 2 & 0 & 1,25 & 0 & 65 & 22,9 & 1,76 \\
\hline
\end{tabular}


(a)

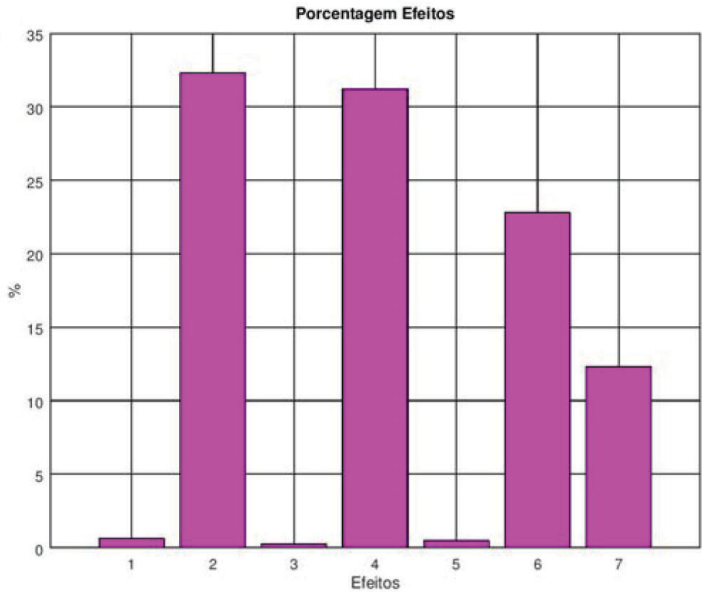

(b)

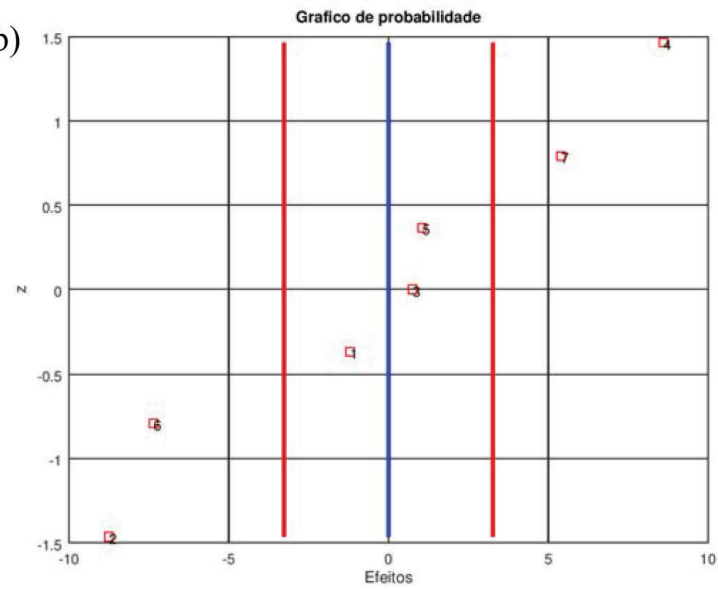

Figura 3. Porcentagem relativa dos contrastes calculados (a) e o gráfico de probabilidade (b) para o exemplo 2

A Figura 3 mostra os gráficos de porcentagem (Figura 3a) e de probabilidade (Figura 3b) para a primeira resposta após a utilização da rotina "fabi_efeito":

[efeito1, porc1]=fabi_efeito $(X, y 1,0.62,4.30)$;

Novamente, os dados de entrada (inputs) 0.62 (erro de um efeito) e 4.30 (valor de $t$ para o erro experimental) podem ser substituídos por zero, caso não tenham sido efetuadas réplicas. Na preparação da matriz $\mathbf{X}$ não é necessário considerar os experimentos no ponto central.

Com os gráficos da Figura 3 é possível perceber que os contrastes mais importantes são o $2+134$ (identificado com o número 2 na Figura 3), 4+123 (número 4), 13+24 (número 6) e 14+23 (número 7). Assim, para obter menores tamanhos de partícula, é necessário utilizar as variáveis 2 e 4 nos níveis alto e baixo, respectivamente. As variáveis 1 e 3 devem estar nos níveis opostos das variáveis 4 e 2 , respectivamente, ou seja, nos níveis alto (variável 1) e baixo (variável 3). Assim, este exemplo permitiu ordenar as variáveis em termos de importância, sendo que as variáveis 2 e 4 mostraram maiores contrastes que as demais sobre a resposta.

O material suplementar (Resultados para a segunda resposta do exemplo 2) mostra os gráficos obtidos para a resposta 2. Os maiores contrastes foram obtidos para a variável 4 (identificada com o número 4), o contraste 12+34 (número 5), a variável 2 (número 2) e por fim o contraste 13+24 (número 6). Para obter maior distribuição do tamanho de partículas é necessário utilizar a variável 4 no nível baixo, as variáveis 1 e 2 devem estar em níveis opostos, a variável 2 deve estar no nível alto (assim a variável 1 deverá estar no nível baixo) e a variável 3 deve estar no nível oposto da variável 1, ou seja, no nível alto.

Ao comparar as conclusões para as 4 variáveis frente às duas respostas, nota-se que as variáveis 1 e 3 apresentam condições contraditórias. Assim, o ponto central poderia ser o nível mais adequado para ambas, permitindo assim a obtenção de uma condição de compromisso para as respostas avaliadas.

\section{Modelos de regressão}

\section{Exemplo 3}

No exemplo 3 voltamos ao artigo utilizado no exemplo 1. Após os resultados apontarem que as variáveis concentrações de $\mathrm{HCl}$ e de $\mathrm{NaBH}_{4}$ foram as mais importantes (etapa 1), a próxima ação foi realizar um planejamento fatorial do tipo Doehlert no sentido de obter uma condição ótima de trabalho. Uma das características do planejamento fatorial do tipo Doehlert ${ }^{42}$ é a possibilidade de testar as variáveis em diferentes números de níveis. No exemplo 1, nota-se que a variável $2\left(\mathrm{NaBH}_{4}\right)$ apresenta um efeito ligeiramente maior que a variável $1(\mathrm{HCl})$. Assim, a concentração de $\mathrm{NaBH}_{4}$ foi testada em 5 níveis (de $-1 \mathrm{a}+1)$ e a concentração de $\mathrm{HCl}$ em 3 (de -0,866 a $+0,866$ ). Os autores poderiam ter optado também pelo planejamento fatorial composto central (etapa 2 da Figura 1), no qual o número de níveis é o mesmo para ambas as variáveis. A Tabela 3 mostra os experimentos efetuados, bem como os sinais monitorados para o analito em questão.

Com os experimentos descritos na Tabela 3, é possível calcular um modelo com 6 coeficientes: b0 (constante), b1, b2 (lineares), b11, b22 (quadráticos) e b12 (interação). Assim, é necessário montar uma matriz $\mathbf{X}$ contendo 9 linhas (os experimentos) e 6 colunas (os coeficientes). O coeficiente b0 é estabelecido por uma coluna com números 1. Já os coeficientes b1 e b2 são as colunas codificadas das duas variáveis mostradas na Tabela 3. Os coeficientes b11 e b22 são os termos quadráticos das variáveis 1 e 2 , respectivamente e são obtidos ao elevar ao quadrado essas duas variáveis. O coeficiente b12 é obtido ao multiplicar as colunas das variáveis 1 e 2 (Tabela 3). Para efetuar os cálculos dos coeficientes de regressão e da tabela Anova (etapas 3 e 4 da Figura 1), foi utilizada a função regression2 (Material suplementar) e a linha de comando foi a seguinte:

[Mod_par,Coef,Pred] = regression2(X,y,48.7,2);

em que X, y, 48.7 e 2 são os parâmetros de entrada (inputs) do modelo compostos pela matriz $\mathbf{X}$, vetor $\mathrm{y}$, soma quadrática do erro puro (SQEP) e seu grau de liberdade, respectivamente. Os parâmetros de saída (outputs) da função regression2 são a tabela Anova (Mod_par), os coeficientes calculados (Coef) e os valores previstos pelo modelo (Pred). A SQEP foi obtida por meio das réplicas efetuadas no ponto central:

$$
S Q E P=\frac{\sum\left(x_{i}-\bar{x}\right)^{2}}{n-1}
$$

em que $x_{i}$ e $\bar{x}$ são as réplicas efetuadas no ponto central e a média dos mesmos, respectivamente.

A rotina "regression2" mostra inicialmente 4 gráficos que são apresentados na Figura 4. O primeiro gráfico (Figura 4a) mostra as médias quadráticas da regressão (MQR, coluna verde), resíduo (MQr, coluna amarela), erro puro (MQEP, coluna vermelha) e falta de ajuste (MQFaJ, coluna rosa). Os números inseridos nas colunas da Figura 4a mostram os valores das médias quadráticas. No caso específico da MQr e MQFaJ são mostrados também os valores de $t$ tabelado com $95 \%$ de confiança. A 
Tabela 3. Planejamento fatorial do tipo Doehlert para otimização das condições experimentais relativas à determinação do $\mathrm{Sb}$

\begin{tabular}{|c|c|c|c|c|c|}
\hline \multirow{2}{*}{ Experimentos } & \multicolumn{2}{|c|}{$\mathrm{HCl}(\mathrm{v} 1)$} & \multicolumn{2}{|c|}{$\mathrm{NaBH}_{4}(\mathrm{v} 2)$} & \multirow{2}{*}{$\begin{array}{c}\text { Intensidade de } \\
\text { fluorescência do } \mathrm{Sb}\end{array}$} \\
\hline & Codificado & Real (mol/L) & Codificado & $\operatorname{Real}(\%, \mathrm{~m} / \mathrm{v})$ & \\
\hline 1 & 0,866 & 5 & 0,5 & 1,8 & 367 \\
\hline 2 & 0,866 & 5 & $-0,5$ & 1,4 & 660 \\
\hline 3 & 0 & 4 & -1 & 1,2 & 762 \\
\hline 4 & $-0,866$ & 3 & $-0,5$ & 1,4 & 787 \\
\hline 5 & $-0,866$ & 3 & 0,5 & 1,8 & 434 \\
\hline 6 & 0 & 4 & 1 & 2,0 & 167 \\
\hline 7 & 0 & 4 & 0 & 1,6 & 651 \\
\hline 8 & 0 & 4 & 0 & 1,6 & 643 \\
\hline 9 & 0 & 4 & 0 & 1,6 & 652 \\
\hline
\end{tabular}

situação desejável é que a MQR seja muito maior que a MQr. Idealmente, a razão entre MQR e MRr deveria ser alto para afirmar que o modelo calculado possui uma adequada condição estatística, permitindo a sua utilização para previsões. ${ }^{48} \mathrm{O}$ cálculo desta razão representa a variância do modelo, em que os valores de Fcalculado e Ftabelado são comparados. Assim, esse parâmetro é uma das verificações a serem feitas por meio da tabela Anova (etapa 3 da Figura 1). Para o caso específico do exemplo 3 , o valor de $\mathrm{F}$ foi da ordem de 400. O valor de F tabelado com 5 (MQR) e 3 (MQr) graus de liberdade é 9,013 com 95\% de confiança e a razão entre o valor calculado e o tabelado é superior a 40. Idealmente essa razão deveria ser superior a 10. O segundo gráfico da Figura 4 mostra esses resultados por meio das colunas azuis (Figura $4 b$ ). A terceira coluna azul (a)

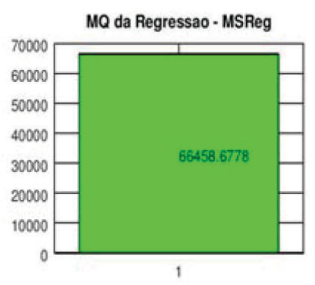

MQ do Erro Puro - MSPE

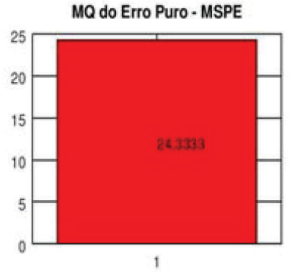

MQ dos residuos - MSres e t

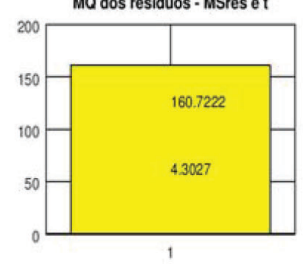

MQ da Falta de Ajuste - MSLoF et

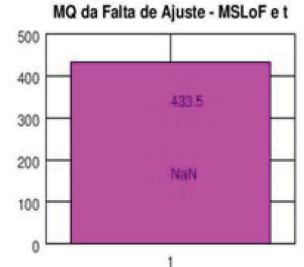

(b)

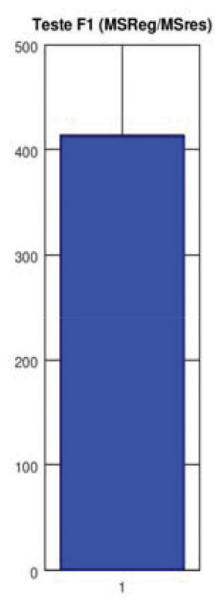

mostra a razão entre os valores de $\mathrm{F}$ (Fcalculado/Ftabelado $=400 / 9=$ cerca de 40). Nessa coluna foi colocada uma linha horizontal vermelha no valor 10 para que o experimentador tenha uma impressão visual da qualidade do modelo.

As primeiras impressões levam a concluir que o modelo calculado apresenta uma boa regressão. Entretanto, é necessário verificar também se o mesmo apresenta ou não falta de ajuste. Essa observação é ilustrada na etapa 4 da Figura 1.

Assim, ao dividir a MQFaJ pela MQEP é obtido um valor de F da ordem de 17. Esse valor calculado deve ser comparado com aquele tabelado. O gráfico com colunas vermelhas da Figura 4 mostra os valores de $\mathrm{F}$ calculado e tabelado e também a razão entre ambos (c)

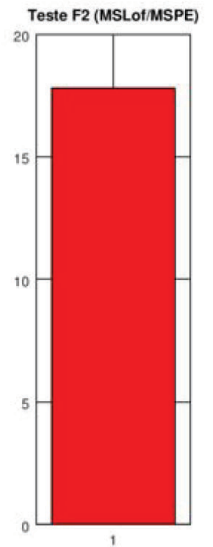

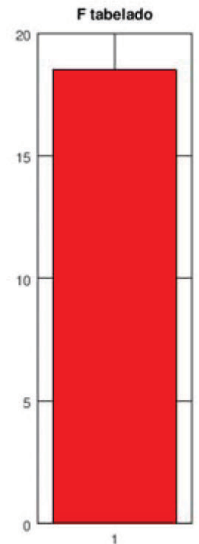

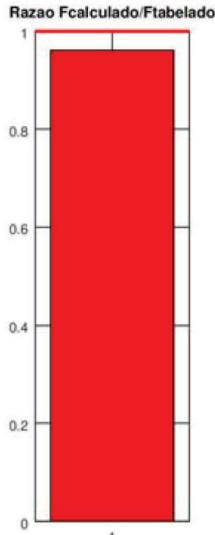

(d)
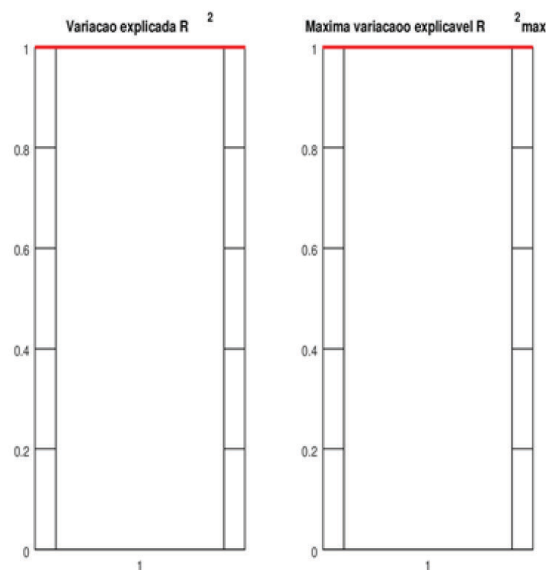

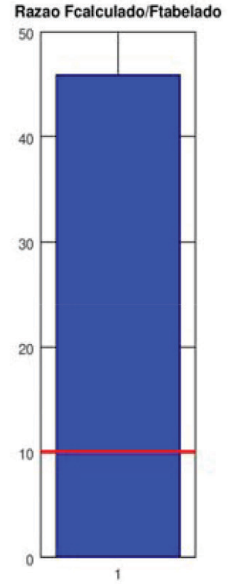

Figura 4. Gráficos dos valores de médias quadráticas (a), teste F para MQR e MQr (b), teste F para MQFaJ e MQEP (c) e variação explicada $\left(R^{2}\right)$ e explicável $\left(R^{2} \max \right)($ d) para o exemplo 3 
(Figura 4c). É possível notar que o valor calculado é inferior ao tabelado com $95 \%$ de confiança. Esse gráfico mostra também que o modelo calculado não apresenta falta de ajuste, pois os valores de MQFaJ e MQEP se confundem estatisticamente (F calculado $<\mathrm{F}$ tabelado). A terceira coluna vermelha mostra a razão entre os valores de $\mathrm{F}($ Fcalculado/Ftabelado $=17 / 19=$ inferior a 1$)$. A linha horizontal vermelha nesta coluna foi colocada no valor 1 para auxiliar o experimentador na verificação da falta de ajuste ou não do modelo.

Por fim, o quarto gráfico da Figura 4 mostra os valores de variação explicada $\left(R^{2}\right)$ e a máxima variação explicável $\left(R^{2} \max \right)^{25}$ (Figura $\left.4 d\right)$. A situação ideal é que ambos sejam o mais próximo de 1 , o que de fato está ocorrendo, visto que ambas as colunas estão próximas às linhas vermelhas horizontais que indicam o valor 1. Essas informações podem ser visualizadas também na matriz Mod_par que é gerada pela rotina "regression2".

Como o modelo calculado não apresenta evidências de falta de ajuste, é possível utilizar a MQr como variância para os 6 coeficientes calculados. Além disso, é necessário informar à rotina computacional o valor de $t$ com n-1 graus de liberdade da MQr. No caso específico do exemplo 3 esses valores serão: 161 e 4,30, respectivamente (ver Figura 4a).

Após a inserção dessas informações são mostrados mais três gráficos, que são apresentados na Figura 5. O primeiro gráfico (Figura 5a) mostra o valor experimental versus o previsto para a propriedade que está sendo modelada (círculos). Além disso, os símbolos (+) e (-) na Figura 5 a mostram os valores quando os coeficientes foram somados e subtraídos ao intervalo de confiança, respectivamente. Assim, o leitor pode ter uma percepção sobre a faixa de valores previstos para um determinado experimento. O segundo gráfico (Figura 5b) mostra os valores previstos versus o resíduo e o histograma dos resíduos. A situação ideal é que os resíduos estejam distribuídos de forma aleatória e próximos de zero. O último gráfico da Figura 5 mostra os coeficientes de regressão (Figura 5c) calculados (triângulos invertidos) e o intervalo de confiança ( intervalo de confiança do sexto coeficiente (b12) compreende o valor zero, sendo considerado não significativo ao nível de confiança de $95 \%$. Para visualizar os coeficientes com mais detalhes basta digitar "Coef" na janela de comandos do Octave, na qual é mostrada uma matriz com 6 linhas (os 6 coeficientes) e 4 colunas para: os coeficientes, os coeficientes subtraídos do intervalo de confiança, os coeficientes somados ao intervalo de confiança e o intervalo de confiança.

Como o coeficiente b12 não é significativo, é necessário recalcular o modelo ${ }^{58}$ utilizando apenas os coeficientes válidos. Entretanto, é necessário visualizar se novas réplicas serão formadas após a eliminação do coeficiente insignificante. No caso específico do exemplo 3 não foram formadas novas réplicas e o seguinte comando deve ser dado no Octave:

[Mod_par,Coef,Pred] = regression2(X(:,1:5),y,48.7,2);

Veja que o coeficiente de número 6 (b12) foi omitido. O material suplementar (Resultados para o exemplo 3 recalculado) mostra os gráficos gerados para o modelo recalculado. $\mathrm{O}$ modelo apresentou uma leve falta de ajuste, mas nesse caso foi ainda utilizada a MQr para calcular a variância dos coeficientes. Os coeficientes válidos com $95 \%$ de confiança foram:

Resposta $=\underset{ \pm 34}{648}-\underset{ \pm 34,2}{56,0} v_{1}-\underset{ \pm 34}{306} v_{2}-\underset{ \pm 54,0}{54,2} v_{1}^{2}-\underset{ \pm 54}{184} v_{2}^{2}$

Ao modelo obtido pode ser aplicada a derivada parcial para calcular a região ótima de trabalho ou ainda pode ser visualizada no gráfico de superfície de resposta. A Figura 6 mostra a superfície de resposta obtida no arquivo "template_superfície" do Excel e nota-se (a)

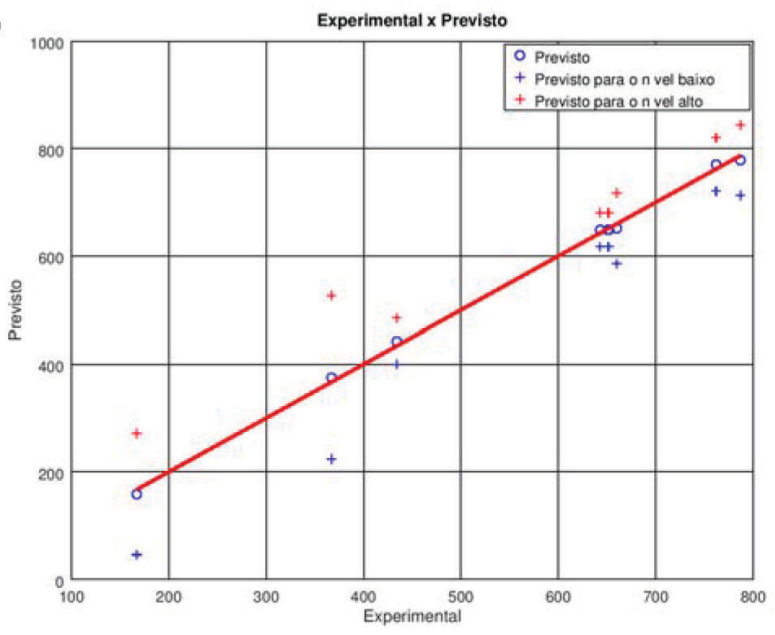

(b)
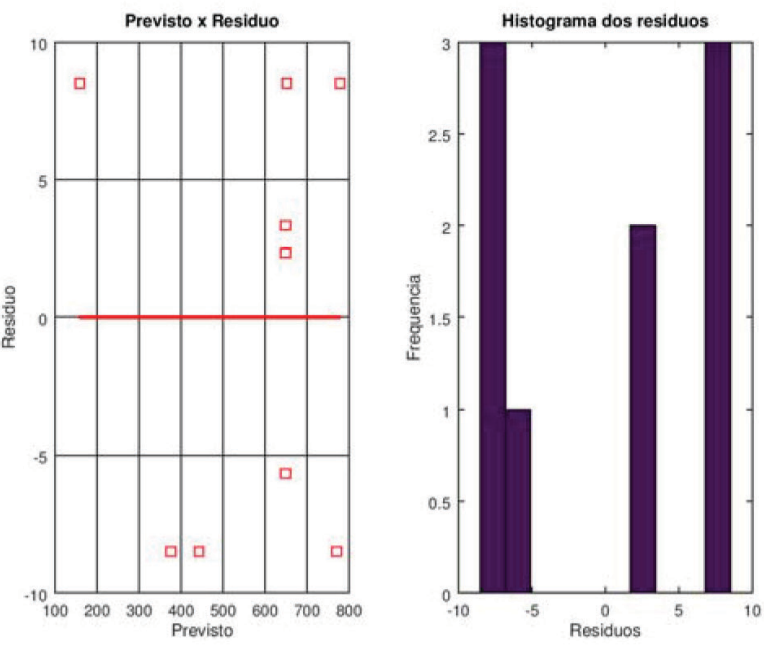

(c)

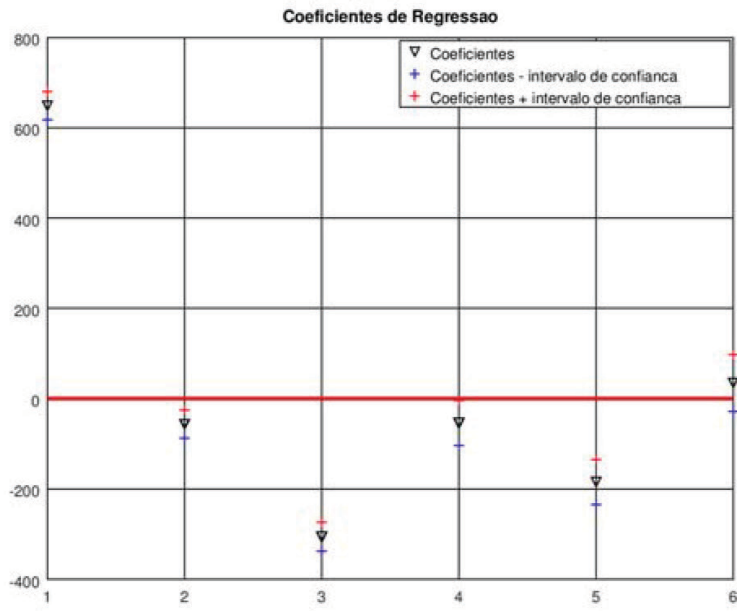

Figura 5. Gráficos dos valores experimental versus previsto (a), previsto versus resíduo e histograma dos resíduos (b) e coeficientes de regressão (c) para o exemplo 3

uma região de trabalho que permite obter sinais de fluorescência mais intensos (Figura 6a).

Esta região pode ser melhor visualizada ao apagar os valores relativos à mesma no template_superfície. Essa operação irá gerar um efeito visual no gráfico de superfície de resposta que é ilustrado também na Figura 6b. A região ótima de trabalho após aplicar a derivada parcial é 3,4 mol/L para o $\mathrm{HCl}(\mathrm{v} 1)$ e $1,3 \% \mathrm{~m} / \mathrm{v}$ para o $\mathrm{NaBH}_{4}$. 
Nesta condição, o sinal de fluorescência previsto é da ordem de 790. Os autores do trabalho do exemplo 3 indicaram uma região de ótimo muito próxima daquela calculada: 3,07 e 1,24 para o $\mathrm{HCl}$ e $\mathrm{NaBH}_{4}$, respectivamente. Nas Figuras 6c a $6 \mathrm{f}$ são mostrados também estes dois pontos por meio das superfícies e dos gráficos de contorno.

Alternativamente, a superfície de resposta pode ser calculada utilizando a função "super_fabi" que foi preparada no Octave. O material suplementar mostra o código da função e a forma como deve ser utilizada está detalhada por meio de vídeo.

\section{Exemplo 4}

O exemplo $4^{56}$ mostra a utilização de um planejamento BoxBehnken (etapa 2 da Figura 1). Os autores investigaram o comportamento de 4 variáveis no rendimento de benzaldeído. A Tabela 4 mostra os 29 experimentos efetuados, bem como a resposta obtida para cada um.
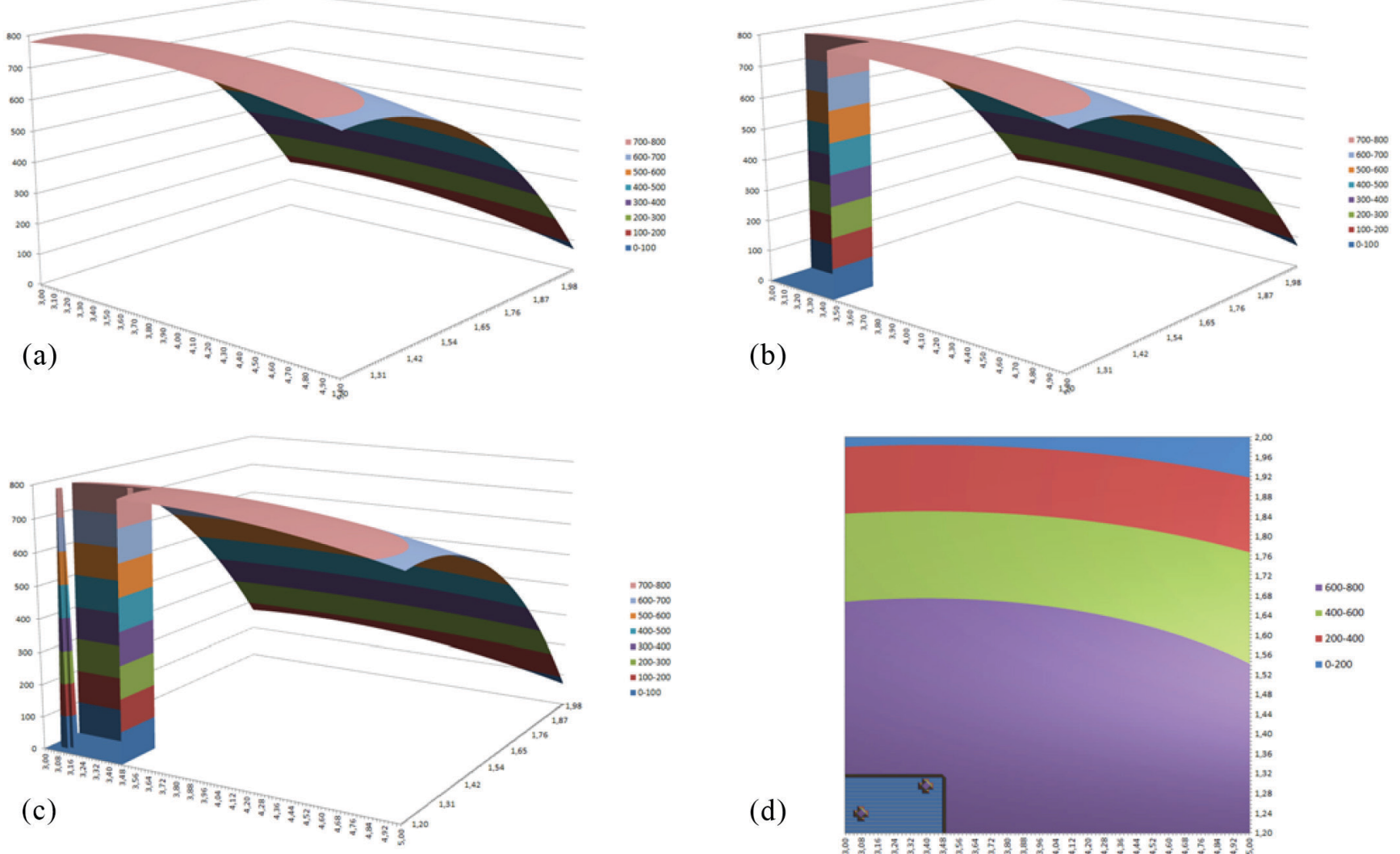
o planejamento fatorial em questão é possível calcular 15 coeficientes: b0 (constante), b1, b2, b3, b4 (coeficientes lineares para cada variável), b11, b22, b33, b44 (coeficientes quadráticos para cada variável), b12, b13, b14, b23, b24, b34 (coeficientes de interação de segunda ordem para as variáveis). Os experimentos de 25 a 29 representam as 5 réplicas no ponto central para calcular a SQEP (ver Equação 6).

O material suplementar (Primeiro modelo calculado) mostra os gráficos gerados ao calcular o modelo e o mesmo não apresentou falta de ajuste:

$$
\begin{aligned}
\text { Resposta }= & \underset{ \pm 1,14}{92,28}+\underset{ \pm 0,73}{6,17} v_{1}+\underset{ \pm 0,73}{2,14} v_{2}+\underset{ \pm 0,73}{2,79} v_{3}+\underset{ \pm 0,73}{1,99} v_{4}- \\
& 5,70 v_{1}^{2}-\underset{ \pm 1,00}{3,41} v_{2}^{2}-\underset{ \pm 1,00}{2,00} v_{3}^{2}-\underset{ \pm 1,00}{2,58} v_{4}^{2}-\underset{ \pm 1,27}{1,32} v_{12}- \\
& 31,00 \\
& 3,15 v_{13}+\underset{ \pm 1,27}{0,19} v_{14}-\underset{ \pm 1,27}{2,34} v_{23}-\underset{ \pm 1,27}{2,49} v_{24}+\underset{ \pm 1,27}{0,22} v_{34}
\end{aligned}
$$

(d)

(b)

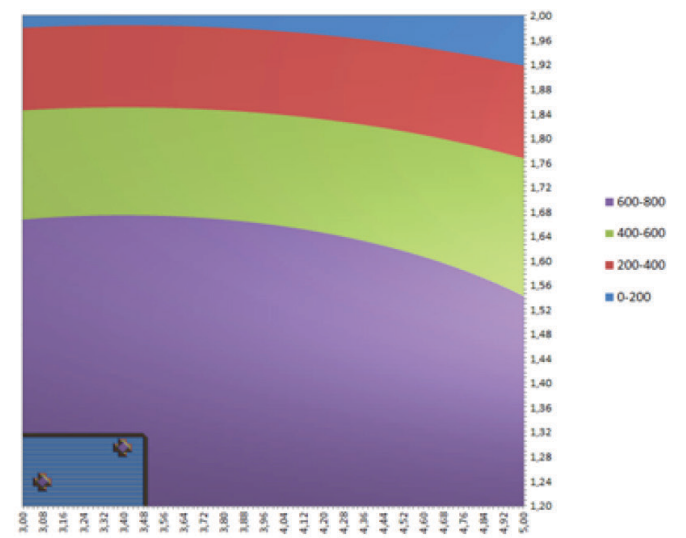

(f)

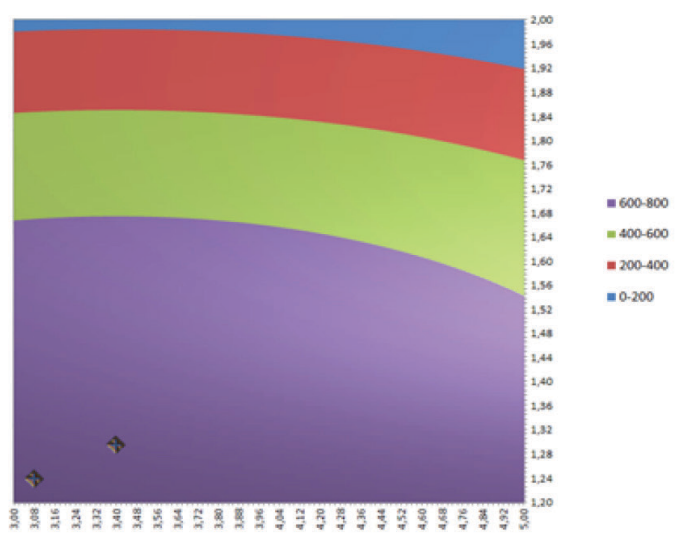

Figura 6. Superfícies de respostas $(a, b, c, e)$ e gráficos de contorno $(d, f)$ para o modelo gerado pelo exemplo 3 
Tabela 4. Planejamento fatorial Box-Behnken para verificar a influência de 4 variáveis no rendimento de produção do benzaldeído.

\begin{tabular}{|c|c|c|c|c|c|c|c|c|c|}
\hline \multirow[t]{2}{*}{ Experimento } & \multicolumn{2}{|c|}{ Massa de catalisador (v1) } & \multicolumn{2}{|c|}{$\begin{array}{l}\text { Concentração de } \mathrm{H}_{2} \mathrm{O}_{2} \text { em } \\
\text { álcool benzílico (v2) }\end{array}$} & \multicolumn{2}{|c|}{ Tempo de reação (v3) } & \multicolumn{2}{|c|}{ Volume de água (v4) } & \multirow{2}{*}{$\begin{array}{c}\text { Rendimento } \\
(\%)\end{array}$} \\
\hline & Codificado & Real (g) & Codificado & Real $(\%)$ & Codificado & Real (h) & Codificado & Real (mL) & \\
\hline 1 & -1 & 0,7 & -1 & 50 & 0 & 4 & 0 & 20 & 73,00 \\
\hline 2 & 1 & 0,9 & -1 & 50 & 0 & 4 & 0 & 20 & 88,15 \\
\hline 3 & -1 & 0,7 & 1 & 75 & 0 & 4 & 0 & 20 & 80,98 \\
\hline 4 & 1 & 0,9 & 1 & 75 & 0 & 4 & 0 & 20 & 90,82 \\
\hline 5 & 0 & 0,8 & 0 & 66 & -1 & 3 & -1 & 15 & 84,55 \\
\hline 6 & 0 & 0,8 & 0 & 66 & 1 & 5 & -1 & 15 & 88,23 \\
\hline 7 & 0 & 0,8 & 0 & 66 & -1 & 3 & 1 & 25 & 86,86 \\
\hline 8 & 0 & 0,8 & 0 & 66 & 1 & 5 & 1 & 25 & 91,41 \\
\hline 9 & -1 & 0,7 & 0 & 66 & 0 & 4 & -1 & 15 & 75,38 \\
\hline 10 & 1 & 0,9 & 0 & 66 & 0 & 4 & -1 & 15 & 88,50 \\
\hline 11 & -1 & 0,7 & 0 & 66 & 0 & 4 & 1 & 25 & 80,24 \\
\hline 12 & 1 & 0,9 & 0 & 66 & 0 & 4 & 1 & 25 & 94,13 \\
\hline 13 & 0 & 0,8 & -1 & 50 & -1 & 3 & 0 & 20 & 80,17 \\
\hline 14 & 0 & 0,8 & 1 & 75 & -1 & 3 & 0 & 20 & 88,09 \\
\hline 15 & 0 & 0,8 & -1 & 50 & 1 & 5 & 0 & 20 & 91,47 \\
\hline 16 & 0 & 0,8 & 1 & 75 & 1 & 5 & 0 & 20 & 90,03 \\
\hline 17 & -1 & 0,7 & 0 & 66 & -1 & 3 & 0 & 20 & 72,34 \\
\hline 18 & 1 & 0,9 & 0 & 66 & -1 & 3 & 0 & 20 & 89,63 \\
\hline 19 & -1 & 0,7 & 0 & 66 & 1 & 5 & 0 & 20 & 84,61 \\
\hline 20 & 1 & 0,9 & 0 & 66 & 1 & 5 & 0 & 20 & 89,31 \\
\hline 21 & 0 & 0,8 & -1 & 50 & 0 & 4 & -1 & 15 & 79,08 \\
\hline 22 & 0 & 0,8 & 1 & 75 & 0 & 4 & -1 & 15 & 88,30 \\
\hline 23 & 0 & 0,8 & -1 & 50 & 0 & 4 & 1 & 25 & 88,02 \\
\hline 24 & 0 & 0,8 & 1 & 75 & 0 & 4 & 1 & 25 & 87,29 \\
\hline 25 & 0 & 0,8 & 0 & 66 & 0 & 4 & 0 & 20 & 91,61 \\
\hline 26 & 0 & 0,8 & 0 & 66 & 0 & 4 & 0 & 20 & 91,70 \\
\hline 27 & 0 & 0,8 & 0 & 66 & 0 & 4 & 0 & 20 & 93,00 \\
\hline 28 & 0 & 0,8 & 0 & 66 & 0 & 4 & 0 & 20 & 92,11 \\
\hline 29 & 0 & 0,8 & 0 & 66 & 0 & 4 & 0 & 20 & 93,00 \\
\hline
\end{tabular}

O primeiro modelo estabelecido mostra que dois coeficientes não são significativos ao nível de confiança de $95 \%$ : b14 $(0,19 \pm$ 1,27 , décimo segundo coeficiente) e b34 (0,22 $\pm 1,27$, décimo quinto coeficiente). A retirada desses dois coeficientes não permitiu a formação de novas réplicas. O modelo foi recalculado na ausência desses coeficientes e os gráficos resultantes são mostrados no Material Suplementar (Modelo recalculado)

O modelo final gerado apresentou os seguintes coeficientes válidos:

$$
\begin{aligned}
\text { Resposta }= & 92,28+\underset{ \pm 0,73}{60,17} v_{1}+\underset{ \pm 0,45}{2,14} v_{2}+\underset{ \pm 0,45}{2,79} v_{3}+\underset{ \pm 0,45}{1,99} v_{4}- \\
& 5,70 v_{1}^{2}-\underset{ \pm 0,41}{3} v_{2}^{2}-\underset{ \pm 0,65}{2,00} v_{3}^{2}-\underset{ \pm 0,65}{2,58} v_{4}^{2}- \\
& \pm 0,65 \\
& 1,32 v_{12}-\underset{ \pm 0,65}{3,15} v_{13}-\underset{ \pm 0,82}{2,34} v_{23}-\underset{ \pm 0,82}{2,49} v_{24} \\
&
\end{aligned}
$$

Por meio da Equação 9 nota-se que todos os coeficientes são significativos ao nível de confiança de $95 \%$. Para identificar a melhor condição de trabalho foi utilizada a ferramenta Solver ${ }^{59}$ disponível no Microsoft Excel. Com o Solver foram testados dois métodos para solução do problema: o Gradação Reduzida Generalizada (GRG) Não Linear e o Evolutionary. Além disso, pode ser aplicada também a derivada parcial. Os cálculos da derivada parcial são mostrados no Octave e o material suplementar (Cálculo da derivada parcial) mostra a realização dos mesmos com o estabelecimento das matrizes.

Os resultados obtidos são mostrados na Tabela 5. Com a utilização do Solver é necessário estabelecer níveis máximos e mínimos para cada uma das variáveis. No caso específico do exemplo 4, a condição ideal para as 4 variáveis deve estar entre -1 e +1 (valores codificados) e representam o domínio experimental testado. A Tabela 5 mostra também a melhor condição operacional identificada pelos autores do

Tabela 5. Melhor condição de trabalho identificada pelo modelo proposto para o exemplo 4

\begin{tabular}{ccccccc}
\hline \multirow{2}{*}{ Método de resolução } & \multicolumn{3}{c}{ Variáveis } & \multicolumn{2}{c}{$\begin{array}{c}\text { Resposta prevista* ou obtida experi- } \\
\text { mentalmente** }\end{array}$} \\
\cline { 2 - 7 } & GRD & 0,84 & 65 & 4,4 & 22,0 & $94,5^{*}$ \\
Solver (Microsoft Excel) & Evolutionary & 0,84 & 65 & 4,4 & 22,0 & $94,5^{*}$ \\
\hline Derivada parcial (Octave) & 0,84 & 62 & 4,4 & 22,0 & $94,5^{*}$ \\
\hline Condição obtida pelos autores & 0,84 & 66 & 4,4 & 22,3 & & $94,0^{* *}$ \\
\hline
\end{tabular}


trabalho. Nota-se que as duas estratégias de resolução da Equação 9 (Solver e derivada parcial) permitem obter resultados muito próximos.

\section{CONCLUSÕES}

A Tabela 6 mostra uma lista dos vídeos elaborados para melhor detalhamento dos cálculos efetuados neste tutorial, bem como, o uso das ferramentas desenvolvidas. Cada exemplo apresenta vídeos curtos que permitem que o leitor acompanhe todas as etapas de utilização do material proposto. No link abaixo é possível efetuar o download dos vídeos, bem como das rotinas computacionais "fabi_efeito", "regression2", "super_fabi" e do "template_superfície": https://www.dropbox.com/sh/aiuvutcknbch5te/AAC-6Bef2WUsJZ-LJP2imh_Ea?dl=0

Tabela 6. Lista dos vídeos preparados para auxiliar na utilização do Octave, Excel, Solver e exemplos ilustrados neste tutorial

\begin{tabular}{ll}
\hline Identificação do vídeo & Descrição \\
\hline Vídeo_intro & Vídeo introdutório sobre o Octave \\
Vídeo_intro_p2 & Vídeo introdutório: montando a matriz X e o vetor y \\
Exemplo1_v1 & Preparação da matriz X e vetor y \\
Exemplo1_v2 & Erro experimental, graus de liberdade e valor de t \\
Exemplo1_v3 & Introdução dos dados no Octave \\
Exemplo1_v4 & Função "fabi_efeito" \\
Exemplo1_v5 & Gráficos de porcentagem e de probabilidade \\
Exemplo2_v1 & Preparação da matriz X e vetor y \\
Exemplo2_v2 & Cálculos para a resposta 1 \\
Exemplo3_v1 & Preparação da matriz X e vetor y \\
Exemplo3_v2 & Soma quadrática do erro puro, SQEP \\
Exemplo3_v3 & Organização dos dados no Octave \\
Exemplo3_v4 & Visualização dos testes F's \\
Exemplo3_v5 & Valores previstos, resíduos e coeficientes \\
Exemplo3_v6 & Modelo recalculado \\
Exemplo3_v7 & Visualização da superfície de resposta ("templa- \\
Exemplo3_v8 & te_superficie” do Excel) \\
Exemplo4_v1 & Visualização da superfície de resposta (função \\
Exemplo4_v2 & Preparação da matriz X, vetor y e SQEP \\
Exemplo4_v3 & Primeiro modelo calculado \\
Exemplo4_v4 & Modelo recalculado \\
Exemplo4_v5 & Introduzindo dados do Octave para o Excel \\
Exemplo4_v6 & Preparação para o Solver \\
Exemplo4_v7 & Identificação da melhor condição de trabalho \\
\hline & Executando derivada parcial no Octave \\
\hline &
\end{tabular}

O material desenvolvido poderá ser aplicável a diversos seguimentos, tais como Quality by design (QbD), Process analytical technology (PAT), planejamento de misturas, e também àqueles que envolvam múltiplas respostas (desejabilidade). ${ }^{60,61}$

Além disso, estas ferramentas podem ser empregadas em métodos da química verde,${ }^{62}$ no cálculo de incertezas, ${ }^{63}$ e também em normas de diversas agências regulatórias, tais como o Instituto Nacional de Metrologia, Qualidade e Tecnologia (Inmetro) e a Agência Nacional de Vigilância Sanitária (Anvisa).

\section{MATERIAL SUPLEMENTAR}

O Material Suplementar está disponível em http://quimicanova. sbq.org.br, em formato pdf com acesso livre. Esse material apresenta os seguintes itens:
- Rotina computacional "fabi_efeito"

- Rotina computacional "regression2"

- Rotina computacional "super_fabi"

- Resultados obtidos para o exemplo 1 após a remoção da variável 3

- Resultados para a segunda resposta do exemplo 2

- Resultado para o exemplo 3 recalculado

- Exemplo 4, primeiro modelo calculado

- Exemplo 4, modelo recalculado

- Cálculo da derivada parcial

\section{AGRADECIMENTOS}

Os autores são gratos à Fundação de Amparo à Pesquisa do Estado de São Paulo (FAPESP, processos 2016/00779-6, 2017/055500 e 2016/01513-0) e ao Conselho Nacional de Desenvolvimento Científico e Tecnológico (CNPq, processos 401074/2014-5 e $305637 / 2015-0)$. Os autores expressam gratidão à PubliSBQ por permitir o uso dos exemplos em caráter ilustrativo neste tutorial.

\section{REFERÊNCIAS}

1. Seasholtz, M. B.; Chemom. Intell. Lab. Syst. 1999, 45, 55.

2. Oberg, T.; J. Chem. Educ. 2006, 83, 1178.

3. Teófilo, R. F.; Reis, E. L.; Reis, C.; Silva, G. A.; Kubota, L. T.; J. Braz. Chem. Soc. 2004, 15, 865.

4. Costa, L. M.; Ferreira, S. L. C.; Nogueira, A. R. A.; Nóbrega, J. A.; J. Braz. Chem. Soc. 2005, 16, 1269.

5. Polonini, H. C.; Oliveira, M. A. L.; Ferreira, A. O.; Raposo, N. R. B.; Grossi, L. N.; Brandão, M. A. F.; J. Braz. Chem. Soc. 2011, 22, 1263.

6. Gontijo, E. S. J.; Oliveira, F. S. D.; Fernandes, M. L.; Silva, G. A.; Roeser, H. M. P.; Friese, K.; J. Braz. Chem. Soc. 2014, 25, 208.

7. Pereira-Filho, E. R.; Poppi, R. J.; Arruda, M. A. Z.; Quim. Nova 2002, $25,246$.

8. Silva, J. A.; Damasceno, B. P. G. L.; Silva, F. L. H.; Madruga, M. S.; Santana, D. P.; Quim. Nova 2008, 31, 1073.

9. Vicentini, F. C.; Figueiredo-Filho, L. C. S.; Janegitz, B. C.; Santiago, A.; Pereira-Filho, E. R.; Fatibello-Filho, O.; Quim. Nova 2011, 34, 825.

10. Silva, J. B. P.; J. Braz. Chem. Soc. 2000, 11, 219.

11. Bortoloti, J. A.; Andrade, J. C.; Bruns, R. E.; J. Braz. Chem. Soc. 2004, $15,241$.

12. Snetsinger, P.; Alkhatib, E.; J. Chem. Educ. 2018, 95, 636.

13. Smith, R. B.; Billingham Jr.; J. Chem. Educ. 1968, 45, 113.

14. Norcross, B. E.; Clement, G.; Weinstein, M.; J. Chem. Educ. 1969, 46, 694.

15. Ryswyk, H. V.; Van Hecke, G. R.; J. Chem. Educ. 1991, 68, 878.

16. Oles, P. J.; J. Chem. Educ. 1998, 75, 357.

17. Lang, P. L.; Miller, B. I.; Nowak, A. T.; J. Chem. Educ. 2006, 83, 280.

18. Bouzidi, N.; Gozzi, C.; J. Chem. Educ. 2008, 85, 1544.

19. Krawczyk, T.; Slupska, R.; Baj, S.; J. Chem. Educ. 2015, 92, 317.

20. Oliveira, R. R.; Neves, L. S.; Lima, K. M. G.; J. Chem. Educ. 2012, 89, 1566.

21. Liguori, L.; J. Chem. Educ. 2017, 94, 465.

22. Colombo, R.; Yariwake, J. H.; Lanza, M. R. V.; Quim. Nova 2014, 37, 535.

23. Meftah, M. E.; Bigan, M.; Blondeau, D.; Chem. Educator 2003, 8, 318.

24. Stolzberg, R. J.; Chem. Educator 2001, 6, 291

25. Pereira Filho, E. R.; Planejamento fatorial em química: maximizando a obtenção de resultados, Edufscar: São Carlos, 2015.

26. Ferreira, S. L. C.; Bruns, R. E.; Ferreira, H. S.; Matos, G. D.; David, J. M.; Brandão, G. C.; da Silva, E. G. P.; Portugal, L. A.; dos Reis, P. S.; Souza, A. S.; dos Santos, W. N. L.; Anal. Chim. Acta 2007, 597, 179.

27. Leardi, R.; Anal. Chim. Acta 2009, 652, 161. 
28. Ferreira, S. L. C.; Korn, M. G. A.; Ferreira, H. S.; Silva, E. G. P.; Araújo, R. G. O.; Amorim, F. A. C.; Souza, A. S.; Macedo, S. M.; Lima, D. C.; Jesus, R. M.; Appl. Spectrosc. Rev. 2007, 42, 475.

29. Sundberg, R.; Chemometr. Intell. Lab. Syst. 1994, 24, 1.

30. Lundstedt, T.; Seifert, E.; Abramo, L.; Thelin, B.; Nystrom, A.; Pettersen, J.; Bergman, R.; Chemometr. Intell. Lab. Syst. 1998, $42,3$.

31. Hanrahan, G.; Lu, K.; Crit. Rev. Anal. Chem. 2006, 36, 3.

32. Nunes, C. A.; Freitas, M. P.; Pinheiro, A. C. M.; Bastos, S. C.; J. Braz. Chem. Soc. 2012, 23, 2003.

33. Langsrud, O.; Ellekjaer, M. R.; Naes, T.; J. Chemometr. 1994, 8, 205.

34. Ferreira, S. L. C.; Bruns, R. E.; Silva, E. G. P.; Santos, W. N. L.; Quintella, C. M.; David, J. M.; Andrade, J. B.; Breitkreitz, M. C.; Jardim, I. C. S. F.; Barros Neto, B.; J. Chromatogr. A 2007, 1158, 2.

35. Hibbert, D. B.; J. Chromatogr. B 2012, 910, 2.

36. Strange, R. S.; J. Chem. Educ. 1990, 67, 113.

37. Tarley, C. R. T.; Silveira, G.; Santos, W. N. L.; Matos, G. D.; Silva, E. G. P.; Bezerra, M. A.; Miró, M.; Ferreira, S. L. C.; Microchem. J. 2009, 92,58

38. Novaes, C. G.; Bezerra, M. A.; Silva, E. G. P.; Santos, A. M. P.; Romão, I. L. S.; Santos Neto, J. H.; Microchem. J. 2016, 128, 331.

39. Dejaegher, B.; Heyden, Y. V.; J. Pharmaceut. Biomed. 2011, 56, 141.

40. Sahu, P. K.; Ramisetti, N. R.; Cecchi, T.; Swain, S.; Patro, C. S.; Panda, J.; J. Pharmaceut. Biomed. 2018, 147, 590.

41. Barros Neto, B.; Scarminio, I. S.; Bruns, R. E.; Quim. Nova 2006, 29 , 1401.

42. Ferreira, S. L. C.; Santos, W. N. L.; Quintella, C. M.; Barros Neto, B.; Bosque-Sendra, J. M.; Talanta 2004, 63, 1061.

43. Bezerra, M. A.; Santelli, R. E.; Oliveira, E. P.; Villar, L. S.; Escaleira, L. A.; Talanta 2008, 76, 965.

44. Candioti, L. V.; Zan, M. M. D.; Cámara, M. S.; Goicoechea, H. C.; Talanta 2014, 124, 123.

45. Ferreira, S. L. C.; Introdução às técnicas de planejamento de experimentos, Vento Leste: Salvador, 2015.

46. Box, G. E. P.; Hunter, J. S.; Hunter, W. G.; Statistics for experimenters design, innovation, and discovery, Wiley: Hoboken, 2005.
47. Myers, R. H.; Montgomery, D. C.; Anderson-Cook, C. M.; Response surface methodology process and product optimization using designed experiments, Wiley: Hoboken, 2009.

48. Barros Neto, B.; Scarminio, I. S.; Bruns, R. E.; Como fazer experimentos, Bookman: Porto Alegre, 2010.

49. Teófilo, R. F.; Ferreira, M. M. C.; Quim. Nova 2006, 29, 338.

50. Breitkreitz, M. C.; Souza, A. M.; Poppi, R. J.; Quim. Nova 2014, 37, 564.

51. Pereira-Filho, E. R.; Pereira, F. M. V.; Lemos, S. G.; Chem. Educator 2009, 14, 200.

52. Novaes, C. G.; Yamaki, R. T.; Paula, V. F.; Nascimento Jr.; B. B.; Barreto, J. A.; Valasques, G. S.; Bezerra, M. A.; Rev. Virtual Quim. 2017, 9,1184

53. http://www.octave.org, acessada em junho 2018.

54. Santos, G. S.; Silva, L. O. B., Santos Júnior, A. F.; Silva, E. G. P., Santos, W. N. L.; J. Braz. Chem. Soc. 2018, 29, 185.

55. Morais, W. A.; Barros Neto, B., Cavalcanti, I. M. F.; Xavier Junior, F. H., Magalhães, N. S. S.; Maciel, M. A. M.; J. Braz. Chem. Soc. 2017, 28, 1494.

56. Xiaoxiang, H.; Yingying, K.; Chunhua, X.; Xiujuan, T.; Qing, C.; Kuiwu, W.; Chin-Te, H.; Li-Lic, L.; Shang-Bin, L.; J. Braz. Chem. Soc. 2018, 29, 88.

57. Zaviska, F.; Drogui, P.; Blais, J-F.; Mercier, G.; Lafrance, P.; J. Hazard. Mater. 2011, 185, 1499.

58. Leonardi, D.; Lamas, M. C.; Olivieri, A. C.; J. Pharmaceut. Biomed. 2008, 48, 802 .

59. http://www.solver.com

60. Costa, N. R.; Lourenço, J.; Pereira, Z. L.; Chemometr. Intell. Lab. Syst. 2011, 107, 234.

61. Guerra, R. R. G.; Martins, F. C. P.; Lima, C. G. S.; Gonçalves, R. H.; Leite, E. R.; Pereira-Filho, E. R.; Schwab, R. S.; Tetrahedron Lett. 2017, $58,903$.

62. Gozálvez, J. M.; García-Díaz, J. C.; J. Chem. Educ. 2006, 83, 647.

63. Chui, Q. S. H.; J. Braz. Chem. Soc. 2007, 18, 424. 